\title{
Vacuum-Free Laminated Top Electrode with Conductive Tapes for Scalable Manufacturing of Efficient Perovskite Solar Cells
}

\author{
Yuchuan Shao, Qi Wang, Qingfeng Dong, Yongbo Yuan and Jinsong Huang* \\ Department of Mechanical and Materials Engineering, University of Nebraska-Lincoln, Lincoln, \\ Nebraska 68588, USA.
}

\begin{abstract}
The efficiency of organometal trihalide perovskites (OTP) solar cells have reached that parity of single crystal silicon, and its nature abundant raw material and solution-process capability promise a bright future for commercialization. However, the vacuum based techniques for metal electrode deposition and additional encapsulation layer increase the cost of the perovskite solar cells dramatically and impede their commercialization process. Here, we report a vacuum-free low temperature lamination technique to fabricate the top electrode by commercial conductive tapes (C-tape). The simple fabrication method yields good quality contact and high efficiency device of $12.7 \%$. The C-tapes also encapsulated the devices effectively, resulting in greatly improved device stability. The combination of lamination of electrodes and encapsulation layers into a single step significantly reduce the cost of device fabrication.
\end{abstract}

Keywords: organometal trihalide perovskite; photovoltaic; vacuum-free; lamination electrode; encapsulation.

\section{Introduction:}

${ }^{*}$ Correspond to: jhuang2@unl.edu 
Organometal trihalide perovskites (OTP) have been demonstrated to have power conversion efficiency (PCE) approaching that of the crystalline silicon solar cells after less than five years of research,[1-7] enabled by the many intriguing optoelectronic properties such as large absorption coefficient[8], direct bandgap[9] and long carrier diffusion length[6,10]. In addition to the high efficiency, OTP based photovoltaic cells can be made at low cost, which is essential for the commercialization, because of the low-cost, nature-abundant raw material[11], and low-cost and scalable techniques to deposit OTP materials.[12-14] Due to the huge demand of very large area solar panels to supply enough energy to power the world, high throughput, continuous manufacture methods such as roll-to-roll (R2R) process have the biggest promise in future manufacture of OTP solar panels.

Similar to organic photovoltaic (OPV) manufacturing, one critical step in R2R manufacturing of OTP panels is the lamination formation of electrodes and encapsulation layers[15,16] However, most reported perovskite solar cells adopt metal electrodes (Au, Ag, Al, etc.) deposited by vacuum based thermal-evaporation or sputtering[1-3,14,17,18] which are expensive and time-consuming. Recently there are some attempts to apply Ni mesh/PEDOT:PSS electrode[19] or carbon nanotube networks as electrodes[20]. Nevertheless, these electrodes need to be fabricated by complicated processes with high cost. Another challenge for the commercialization of OTP solar panels is the low resistance of OTPs to moisture. To prolong the lifetime of OTP solar cells, additional encapsulation layers such as epoxy sealant[21] or carbon nanotube/polymer composites[22] are needed to prevent the permeation of the moisture, which, again, increases the fabrication and material cost greatly.

In the manuscript, we report a vacuum-free, one-step, low temperature lamination process to form top electrodes using commercial conductive tapes (C-tapes) in planar heterojunction 
(PHJ) perovskite solar cells which is compatible with future roll-to-roll fabrication process. The simple fabrication method yields good quality contact and thus high efficiency devices above $12 \%$. The laminated electrode effectively encapsulated the perovskite layer and enhanced the device stability.

\section{Results and discussion}

The schematic diagram of the lamination process is illustrated in Fig 1a. Commercial copper $(\mathrm{Cu}) \mathrm{C}$-tapes from TED PELLA Inc. were applied for the lamination formation of the top electrodes. The $\mathrm{C}$-tape consists of $\mathrm{Cu}$ foil backing and conductive acrylic adhesive with micrometer size $\mathrm{Ni}$ particles embedded. The $\mathrm{C}$-tapes were heated to $70{ }^{\circ} \mathrm{C}$ so that the acrylic adhesive was softened to avoid damaging the active layer during the lamination process. A smooth glass rod rolled over the C-tape to attach it onto the photoactive layer. Proper pressure was applied on the C-tapes to remove air-bubbles without damaging the perovskite thin films. The $\mathrm{CH}_{3} \mathrm{NH}_{3} \mathrm{PbI}_{3}\left(\mathrm{MAPbI}_{3}\right)$ layers were fabricated by the interdiffusion of lead iodide $\left(\mathrm{PbI}_{2}\right)$ and a methyl ammonium iodide (MAI) stacking layer followed by a solvent-annealing process[23], which were developed by our group to form smooth, pin-hole free perovskite thin films with average grain size larger than film thickness[24]. The laminated devices had a simplified structure of indium tin oxide (ITO)/poly(3,4-ethylenedioxythiophene) poly(styrenesulfonate) (PEDOT:PSS, 25 nm)/ MAPbI 3 (550 600 nm)/phenyl-C61-butyric acid methyl ester (PCBM, 30 $\mathrm{nm}) /$ C-tape. The laminated devices do not contain $\mathrm{C}_{60}(20 \mathrm{~nm}) / 2,9$-dimethyl-4,7-diphenyl-1,10phenanthroline $(\mathrm{BCP}, 8 \mathrm{~nm}) /$ aluminum $(\mathrm{Al}, 100 \mathrm{~nm})$ layers, unlike the typical devices in our previous studies with $\mathrm{C}_{60} / \mathrm{BCP} / \mathrm{Al}$ stacking layers deposited by thermal vacuum evaporation. Here the devices with the structure of ITO/ PEDOT:PSS/ Perovskite/ $\mathrm{PCBM} / \mathrm{C}_{60} / \mathrm{BCP} / \mathrm{Al}$ are 
referred as regular devices. Therefore, compared to the regular devices, both time and energy consumed to fabricate these laminated OTP solar cells were dramatically reduced.

It is essential to apply a proper pressure on the glass rod to remove all the air bubbles below the $\mathrm{C}$-tape and form high quality contact without impairing the photoactive layer. A systematic study was conducted to find out the optimal pressure. Fig $1 \mathrm{~b}$ shows the current density $(J)$ - voltage $(V)$ curves of the several laminated devices formed under typical pressures of $490 \mathrm{~Pa}, 980 \mathrm{~Pa}$, and $1470 \mathrm{~Pa}$. The variation on devices' parameters, short circuit current density $\left(J_{\mathrm{SC}}\right)$, open circuit voltage $\left(V_{\mathrm{OC}}\right)$, fill factor $(\mathrm{FF}), \mathrm{PCE}$, series resistance $\left(R_{\mathrm{S}}\right)$ and shunt resistance $\left(R_{\mathrm{sh}}\right)$ with different applied pressures are summarized in Fig 1c. The devices with the highest PCE of $12.7 \%$ was fabricated by applying about 980 Pa pressure on the C-tape during the lamination process. And the champion device shows excellent diode behavior with a rectification ratio of about $10^{5}$ at $\pm 1.5 \mathrm{~V}$ (Fig 1d), which is comparable to the best devices with thermallyevaporated metal electrodes. The PCE statistics for 15 laminated $\mathrm{MAPbI}_{3}$ solar cells under the optimized pressure is shown in the Fig 1e. The best PCE reached 12.7\%, and average PCE was $11.4 \%$. The champion device shows small photocurrent hysteresis with different scanning directions and steady PCE output for longer than 10 mins. These results are shown in Fig S1 and S2, respectively. Both too small and too large pressure impair the device performance, but in different ways. Too small pressure, $490 \mathrm{~Pa}$ here, may not completely remove all the air bubbles between the C-tape and the active layer. Thus the effective device working area is smaller than the electrode area, which explains the much smaller $J_{\mathrm{sc}}$ of $12.1 \mathrm{~mA} / \mathrm{cm}^{2}$. The large series resistance in these devices also indicates the poor electrical contact caused by the insufficient pressure. On the other hand, too high pressure also impairs the performance of the lamination solar cells. As shown in the Fig 2c, the shunt resistance decrease dramatically from $1515 \Omega \mathrm{cm}^{2}$ 
to $101 \Omega \mathrm{cm}^{2}$ for the device laminated under too high pressure, which indicates that the C-tapes penetrated the PCBM layer and impaired the photoactive layer.

The formation of excellent mechanical and electrical contact between the active layer and the C-tape were confirmed by the mechanical and electronic testing. Kapton tapes were used to examine the mechanical contact strength by peeling off them. The Kapton tapes were first pasted onto the C-tapes in the laminated devices and the thermally-evaporated $\mathrm{Al}$ electrodes in the regular devices, respectively. As shown in the Fig. 2a, the C-tapes remained intact after removing the Kapton tapes (semitransparent yellow strip), while the thermally-evaporated Al electrodes were easily peeled-off by the Kapton tapes. It indicates that the mechanical strength of the contact between the photoactive layer and the C-tapes is stronger than that between the photoactive layer and the thermally-evaporated $\mathrm{Al}$ electrodes. To examine the electronic contact of the $\mathrm{Cu}$ C-tape with the active layer, photoluminescence (PL) and electroluminescence (EL) (by applying a small voltage on the devices) were performed to map the contact quality. The unique visible emission from $\mathrm{MAPbI}_{3}$ allows us to directly examine the contact quality using optical microscopy or a regular imaging device such as a digital camera. Figure $2 \mathrm{~b}$ shows the PL and EL images of a same laminated finger taken from the ITO side. The areas that are not covered by the C-tape show strong PL emission. It is clear that the area with good contact has weak PL, which can be explained by the efficient charge collection or quenching by the C-tape cathode. Strong EL emission was also observed from the laminated devices from the laminated area, which can be ascribed to the good electron injection from the C-tape to PCBM layer, indicating good electronic contact formed. The smaller EL area than PL area in the images might be caused by the low EL efficiency and low sensitivity of the digital camera used. Nevertheless, there were matched spots which had strong PL but weak or weak EL in the laminated area, as 
shown in the figure. The excellent complimentary emission from PL and EL in these spots indicates that these spots has bad mechanical and electronic contact. This might be caused by our non-optimized lamination process yet using a manual-controlled lamination process, which can be solved using a commercial laminator with much better control of pressure uniformity in the future. To the best of our knowledge, this is the first time that combination of PL and EL mappings were applied to study OTP solar cells.

It is noted that PCE of the laminated perovskite solar cells are still lower than that of the regular devices with thermally-evaporated electrodes which have an average efficiency of $14.5 \%[23]$. To further enhance the performance of the laminated perovskite solar cells, the mechanisms need to be understood that causing the efficiency discrepancy between the laminated devices and regular devices. As mentioned, one apparent difference between them is that the regular devices contain thermally-evaporated $\mathrm{C}_{60} / \mathrm{BCP}$ layer while the laminated devices do not. According to our previous study, $\mathrm{C}_{60}$ and PCBM have complementary passivation effect to OTPs by reduce the trap density of states (tDOS)[25]. Thus we used the thermal admittance spectroscopy (TAS) to examine the difference of the tDOS in the laminated devices and regular devices. As shown in Fig 3a, tDOS of laminated devices are 1 to 2 order of magnitude higher than that of regular devices between the trap depth of 0.40 and $0.47 \mathrm{eV}$, which explains the inferior device performance. However, our previous study show that PCBM also has passivation effect within this trap depth range[25]. It indicates that the PCBM layer might be more or less impaired by the lamination process. The increase of tDOS is expected to decrease the charge carrier recombination lifetime, which is confirmed by the impedance spectroscopy measurement (IS), as shown in the Fig 3b. The devices are always kept under one sun illumination during the IS measurements. The equivalent circuit used to fit the results and the equation to extract the 
recombination lifetime are shown in Figure S1. The carrier recombination lifetime in the laminated devices reduced to half that of the thermally-evaporated devices.

The function of PCBM in the devices was studied by comparing the laminated devices with and without PCBM. As shown in the Fig 3c, the $V_{\mathrm{OC}}$ of the laminated solar cells only decreased by $2.3 \%$ (from $0.88 \mathrm{~V}$ to $0.86 \mathrm{~V}$ ) after removing the PCBM layer. However, the $J_{\text {sc }}$ and $F F$ decreased dramatically, which indicates that the PCBM layer did play an important role in enhancing the charge extraction efficiency of laminated devices. The PL intensity measured under open circuit and short circuit conditions were used to characterize the charge extraction efficiency. In this measurement, the excitation $532 \mathrm{~nm}$ green laser came into the devices from ITO side. When the devices work under the open circuit condition, the photogenerated charge carriers cannot be extracted out and then have to recombine in the devices, yielding strong PL. Under short circuit condition, most of the photogenerated charge carriers can be extracted out as short circuit current, and thus the PL intensity reduces. Therefore when the charge collection efficiency increase, more charges will be extracted out under short circuit condition and $\mathrm{R}$ will increase. Here, from the PL intensity ratio (R) of the devices under open-circuit to short-circuit conditions, we can derive the charge extraction efficiency of devices with and without PCBM layers. As shown in the Fig. 3d, PL quenching was much strong in the device with PCBM, and R increased from 6.7 to 16.7 after inserting the PCBM layer. The results agree with previous study that using fullerenes as electron acceptor[26] for perovskite solar cells can effectively enhance charge extraction efficiency. It is also noted that the device shunt resistance greatly increase from $143 \Omega \mathrm{cm}^{2}$ to $1515 \Omega \mathrm{cm}^{2}$ with PCBM layer. It is thus speculated that PCBM layer can also reduce the damage to the perovskite layers during the lamination process. 
A very important function of the laminated $\mathrm{Cu} C$-tape as top electrodes is to improve the stability of perovskite solar cells because the thick $(0.04 \mathrm{~mm})$ and compact $\mathrm{Cu}$ foil can effectively block the permeation of moisture and oxygen to the photoactive layer. As shown in Fig 4a-b, the laminated perovskite solar cells were more stable than the devices with $100 \mathrm{~nm}$ thermally-evaporated $\mathrm{Al}$ electrodes, no matter in the nitrogen $\left(\mathrm{N}_{2}\right)$ or air environment. The PCE of the laminated perovskite solar cells only decreased slightly from $12.5 \%$ to $11.9 \%$, and from $12.3 \%$ to $11.0 \%$ in $\mathrm{N}_{2}$ filled glovebox and air environment (humidity $>15 \%$ ) after 10 days storage, while the devices with thermally-evaporated electrode (Al, $100 \mathrm{~nm}$ ) degraded seriously from $14.5 \%$ to $8.2 \%$ and from $14.6 \%$ to $3.4 \%$ in $\mathrm{N}_{2}$ and in air. It demonstrated the encapsulation function of C-tapes by inhibiting moisture permeation. The stability of laminated perovskite solar cell is comparable to or even better than the devices with carbon back contact[27] as encapsulation layer or mixed halide perovskite[28]. The optical and PL imaging study gave direct evidence for the effective protection of the perovskite layer by the laminated C-tape. Here we fabricated a laminated finger and a thermally-evaporated finger on a same perovskite thin film. It can be seen from the microscopy image (Fig 4c) that there were many dark dots or cracks on the perovskite film in both uncovered and $\mathrm{Al}$ electrode covered areas after exposing the device to air for 48 hours, which indicates moisture penetrated the thin Al electrodes and cause the degradation of the underneath perovskite layer. On the other hand, the perovskite film beneath the $\mathrm{C}$-tape finger remained intact. The contrast is much stronger from the PL image: most areas showed strong PL with non-uniform distribution, while the PL of perovskite layer beneath C-tape remained uniformly quenched.

\section{Conclusion}


In summary, we demonstrate a facile lamination process to form top electrodes in PHJ perovskite solar cells which enabled vacuum-free fabrication with low-temperature solutionprocess. The C-tapes also encapsulated the devices effectively, resulting in greatly improved device stability. The application of $\mathrm{C}$-tape as top electrode enables the combination of lamination of encapsulation layers and electrodes into a single step, which should significantly reduce the cost of device fabrication. The commercial C-tape used in this study was not optimized yet. Future study needs to explore conductive tape with softer conductive acrylic adhesive or with more uniform Ni particles.

\section{Methods}

Film formation and device fabrication. PEDOT:PSS (Baytron-P 4083, used as received) was spun on a cleaned ITO substrate at a speed of 3000 rounds per minute (rpm). The film was then annealed at $110{ }^{\circ} \mathrm{C}$ for $20 \mathrm{~min} . \mathrm{PbI}_{2}$ and MAI were dissolved into dimethylformamide (DMF) and 2-propanol with concentrations of $650 \mathrm{mg} / \mathrm{ml}$ for $\mathrm{PbI}_{2}$ and $75 \mathrm{mg} / \mathrm{ml}$ for MAI, respectively. Both solutions were pre-heated at $100{ }^{\circ} \mathrm{C}$ for around 10 min to ensure both $\mathrm{MAI}$ and $\mathrm{PbI}_{2}$ were fully dissolved. The $\mathrm{PbI}_{2}$ solution was spun on a PEDOT:PSS layer at 6,000 rpm for $30 \mathrm{~s}$. Then the $\mathrm{PbI}_{2}$ film was transferred onto a $70{ }^{\circ} \mathrm{C}$ hot plate for quick drying. The MAI solution was then spun on top of dried $\mathrm{PbI}_{2}$ film at $6,000 \mathrm{rpm}$ for $30 \mathrm{~s}$ at room temperature to achieve films with thicknesses ranging from 550-600 $\mathrm{nm}$. The spin coated $\mathrm{PbI}_{2} / \mathrm{MAI}$ stacking films were annealed at $100{ }^{\circ} \mathrm{C}$ for 2 hours. After they were cooled down to room temperature, PCBM (in dichlorobenzene, $2 \mathrm{wt} \%$ ) was spun on top of the formed perovskite layers (for the devices with 
PCBM) and the film was annealed at $100{ }^{\circ} \mathrm{C}$ for 45 min to drive the diffusion of PCBM into the perovskite layer. The solar cells were finished by the lamination process to attach the conductive tapes onto the PCBM layer (for devices with PCBM) or the bare perovskite layer (for devices without PCBM). The conductive tapes were purchased from Ted Pella, Inc and were used as received. The sheet resistance and $\mathrm{Z}$ direction resistance of the conductive tapes is $0.001 \mathrm{ohms} / \mathrm{sq}$ and 0.005 ohms $/ \mathrm{in}^{2}$, respectively. The device area is determined by the overlap of the ITO substrate and conductive tapes $\left(10 \mathrm{~mm}^{2}\right.$ on average $)$.

Device characterizations. Simulated AM 1.5G irradiation $\left(100 \mathrm{~mW} / \mathrm{cm}^{2}\right)$ produced by a Xenonlamp-based solar simulator (Oriel 67005, $150 \mathrm{~W}$ Solar Simulator) was used for current-voltage measurement. The light intensity was calibrated by a silicon (Si) diode (Hamamatsu S1133) equipped with a Schott visible-color glass-filtered (KG5 color-filtered). The PL spectra were measured by iHR320 Photoluminescence Spectroscopy at room temperature. A $532 \mathrm{~nm}$ green laser with an intensity of $10 \mathrm{~mW} / \mathrm{cm}^{2}$ from Laserglow Technologies was used as the excitation source in PL measurements. The excitation light incidented to the devices from the ITO side. The thermal admittance spectroscopy was performed using an E4980A Precision LCR Meter from Agilent at frequencies between 0.1 to $1000 \mathrm{kHz}$. Impedance spectroscopy was recorded by the E4980A Precision LCR Meter from Agilent with homemade software. The optical and PL images were taken by locating the samples under an optical microscope (Olympus BX61), which has a high resolution CCD camera integrated (Photometrics, CoolSNAP-cf). The optical microscope worked at reflection mode with the samples illuminated from the topside. 
Acknowledgement: This work was supported by the National Science Foundation under Awards ECCS-1201384 and ECCS-1252623, and Department of Energy under Award DEEE0006709.

\section{Reference}

[1] Zhou, H., Q. Chen, G. Li, S. Luo, T.-b. Song, H.-S. Duan, Z. Hong, J. You, Y. Liu, and Y. Yang, Science 345 (2014) 542-546.

[2] Burschka, J., N. Pellet, S.-J. Moon, R. Humphry-Baker, P. Gao, M.K. Nazeeruddin, and M. Grätzel, Nature 499 (2013) 316-319.

[3] Liu, M., M.B. Johnston, and H.J. Snaith, Nature 501 (2013) 395-398.

[4] Lee, M.M., J. Teuscher, T. Miyasaka, T.N. Murakami, and H.J. Snaith, Science 338 (2012) 643-647.

[5] Abrusci, A., S.D. Stranks, P. Docampo, H.-L. Yip, A.K.-Y. Jen, and H.J. Snaith, Nano letters 13 (2013) 3124-3128.

[6] Stranks, S.D., G.E. Eperon, G. Grancini, C. Menelaou, M.J. Alcocer, T. Leijtens, L.M. Herz, A. Petrozza, and H.J. Snaith, Science 342 (2013) 341-344.

[7] Lim, K.G., H.B. Kim, J. Jeong, H. Kim, J.Y. Kim, and T.W. Lee, Advanced Materials 26 (2014) 6461-6466.

[8] Kojima, A., K. Teshima, Y. Shirai, and T. Miyasaka, Journal of the American Chemical Society 131 (2009) 6050-6051.

[9] Kim, H.-S., C.-R. Lee, J.-H. Im, K.-B. Lee, T. Moehl, A. Marchioro, S.-J. Moon, R. Humphry-Baker, J.-H. Yum, and J.E. Moser, Scientific reports 2 (2012).

[10] Xing, G., N. Mathews, S. Sun, S.S. Lim, Y.M. Lam, M. Grätzel, S. Mhaisalkar, and T.C. Sum, Science 342 (2013) 344-347.

[11] Green, M.A., A. Ho-Baillie, and H.J. Snaith, Nature Photonics 8 (2014) 506-514.

[12] Baikie, T., Y. Fang, J.M. Kadro, M. Schreyer, F. Wei, S.G. Mhaisalkar, M. Graetzel, and T.J. White, Journal of Materials Chemistry A 1 (2013) 5628-5641.

[13] Stoumpos, C.C., C.D. Malliakas, and M.G. Kanatzidis, Inorganic chemistry 52 (2013) 90199038.

[14] Conings, B., L. Baeten, C. De Dobbelaere, J. D'Haen, J. Manca, and H.G. Boyen, Advanced Materials 26 (2014) 2041-2046.

[15] Lee, T.W., J. Zaumseil, S.H. Kim, and J.W. Hsu, Advanced Materials 16 (2004) 2040-2045.

[16] Lee, T.-W., J. Zaumseil, Z. Bao, J.W. Hsu, and J.A. Rogers, Proceedings of the National Academy of Sciences of the United States of America 101 (2004) 429-433.

[17] Heo, J.H., S.H. Im, J.H. Noh, T.N. Mandal, C.-S. Lim, J.A. Chang, Y.H. Lee, H.-j. Kim, A. Sarkar, and M.K. Nazeeruddin, Nature photonics 7 (2013) 486-491.

[18] Kim, H.-S., J.-W. Lee, N. Yantara, P.P. Boix, S.A. Kulkarni, S. Mhaisalkar, M. Gra $\square$ tzel, and N.-G. Park, Nano letters 13 (2013) 2412-2417.

[19] Bryant, D., P. Greenwood, J. Troughton, M. Wijdekop, M. Carnie, M. Davies, K. Wojciechowski, H.J. Snaith, T. Watson, and D. Worsley, Advanced Materials 26 (2014) 7499-7504. 
[20] Li, Z., S.A. Kulkarni, P.P. Boix, E. Shi, A. Cao, K. Fu, S.K. Batabyal, J. Zhang, Q. Xiong, and L.H. Wong, ACS nano 8 (2014) 6797-6804.

[21] Malinkiewicz, O., A. Yella, Y.H. Lee, G.M. Espallargas, M. Graetzel, M.K. Nazeeruddin, and H.J. Bolink, Nature Photonics 8 (2014) 128-132.

[22] Habisreutinger, S.N., T. Leijtens, G.E. Eperon, S.D. Stranks, R.J. Nicholas, and H.J. Snaith, Nano letters 14 (2014) 5561-5568.

[23] Xiao, Z., C. Bi, Y. Shao, Q. Dong, Q. Wang, Y. Yuan, C. Wang, Y. Gao, and J. Huang, Energy \& Environmental Science (2014).

[24] Xiao, Z., Q. Dong, C. Bi, Y. Shao, Y. Yuan, and J. Huang, Advanced Materials 26 (2014) 6503-6509.

[25] Shao, Y., Z. Xiao, C. Bi, Y. Yuan, and J. Huang, Nature communications 5 (2014).

[26] Jeng, J.Y., Y.F. Chiang, M.H. Lee, S.R. Peng, T.F. Guo, P. Chen, and T.C. Wen, Advanced Materials 25 (2013) 3727-3732.

[27] Mei, A., X. Li, L. Liu, Z. Ku, T. Liu, Y. Rong, M. Xu, M. Hu, J. Chen, and Y. Yang, Science 345 (2014) 295-298.

[28] Noh, J.H., S.H. Im, J.H. Heo, T.N. Mandal, and S.I. Seok, Nano letters 13 (2013) 1764-1769.

\section{Figure Legends}

Figure 1. (a) Device structure and schematic of the R2R lamination process. Inset is the photograph of the $\mathrm{Cu}$ conductive tape used; (b) $J-V$ curves of the laminated perovskite solar cells fabricated by different pressure; (c) Variation of the devices' parameters with pressure, $J_{\mathrm{SC}}, V_{\mathrm{OC}}$, FF, PCE, $R_{\mathrm{s}}$ and $R_{\mathrm{sh}}$; (d) Darkcurrent of an optimized laminated devices and a regular device. (e) The statistics of the PCE of 15 laminated perovskite solar cells.

Figure 2. (a) Photograph of (1) a device with Kapton tape on a thermally-evaporated Al electrode, (2) the device after peeling of the Kapton tape, (3) a laminated device with Kapton tape on the $\mathrm{Cu}$ C-tape fabricated with $980 \mathrm{~Pa}$ pressure, (4) the laminated device after peeling off 
the Kapton tape. (b) Photoluminescence and electroluminescence mapping for the same laminated finger, white dash rectangle indicate the area was covered by C-tape.

Figure 3. (a) Trap density of states of laminated devices and regular devices measured by thermal-admittance-spectroscopy; (b) Charge carrier recombination lifetime at different bias measured by impedance spectroscopy; (c) $J-V$ curves of the laminated perovskite solar cells with and without PCBM layer; (d) PL of the laminated perovskite solar cells with PCBM (open red triangles) and without PCBM (open black triangles) at open circuit condition, and with PCBM (solid red triangles) and without PCBM (solid black triangles) at short circuit condition. PL intensities were normalized by the intensities at open circuit conditions.

Figure 4. (a) PCE of devices with laminated and thermally-evaporated electrodes after 240 hours storage in $\mathrm{N}_{2}$ glovebox (a), and in air (b). The optical (c) and PL (d) images of the two different fingers on one perovskite thin film with conductive tape and thermally-evaporated Al electrodes. 
(a)

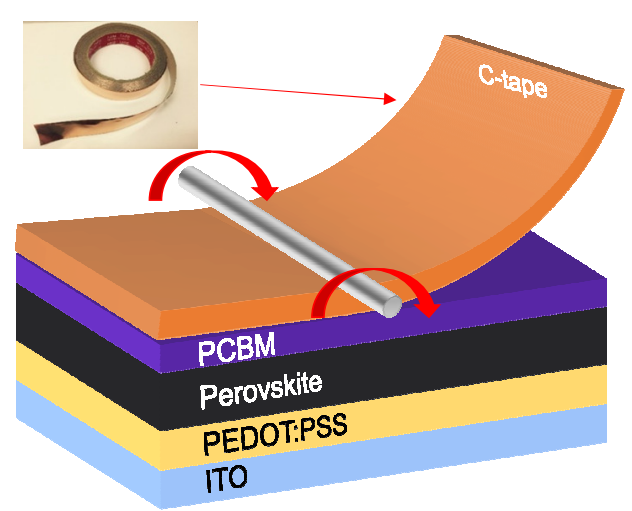

(b)

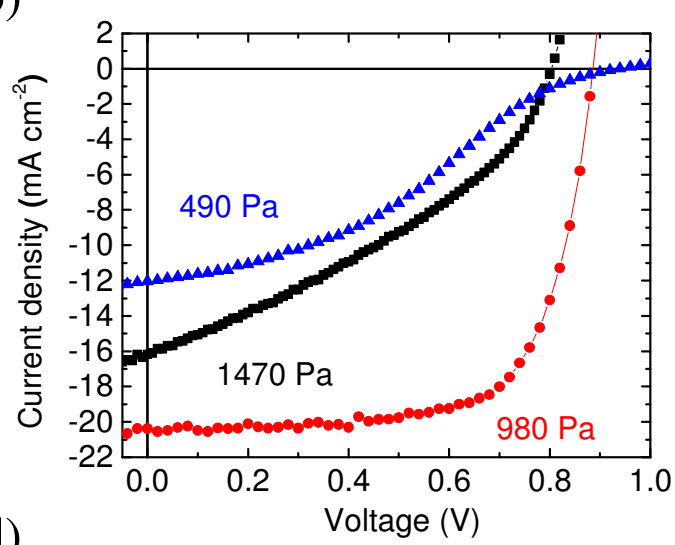

(d)

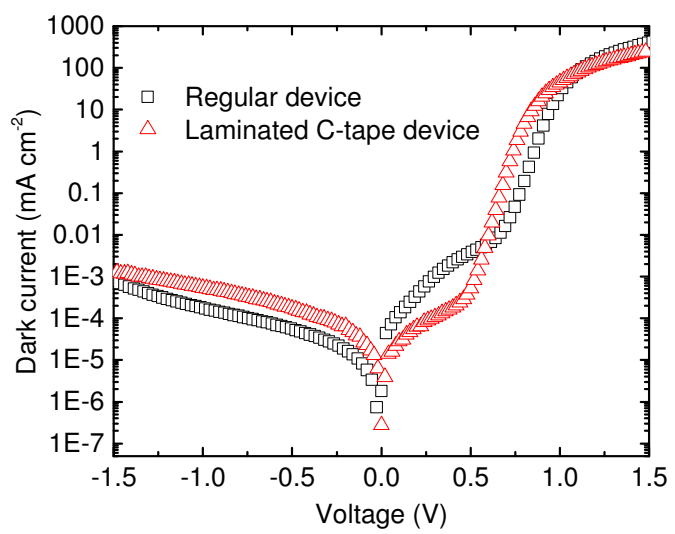

(c)

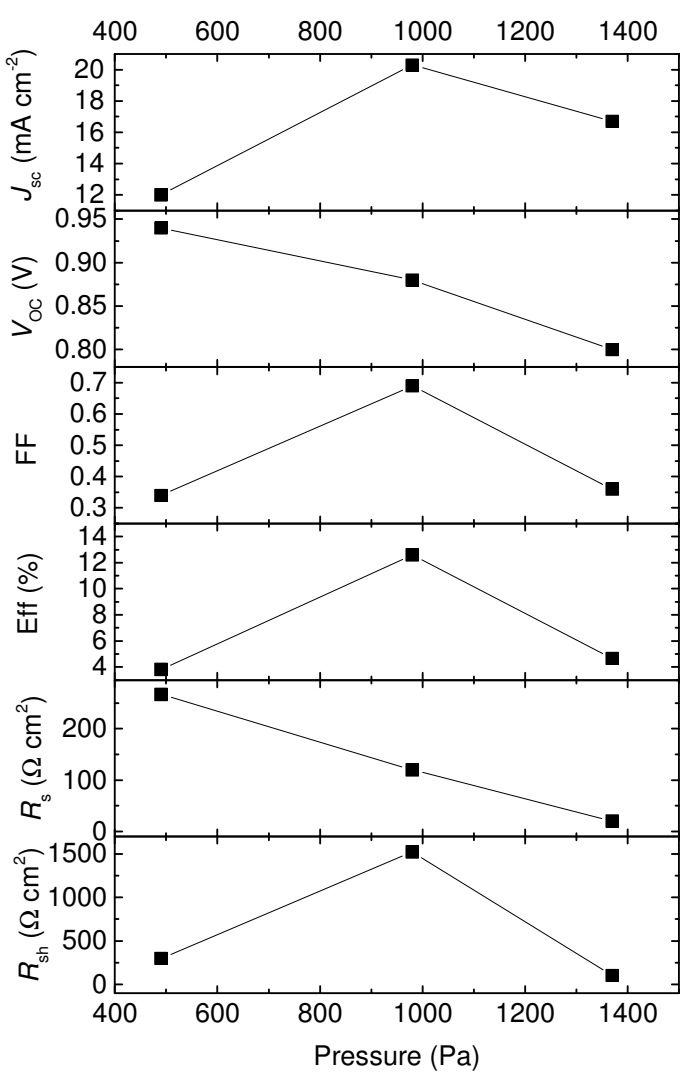

(e)

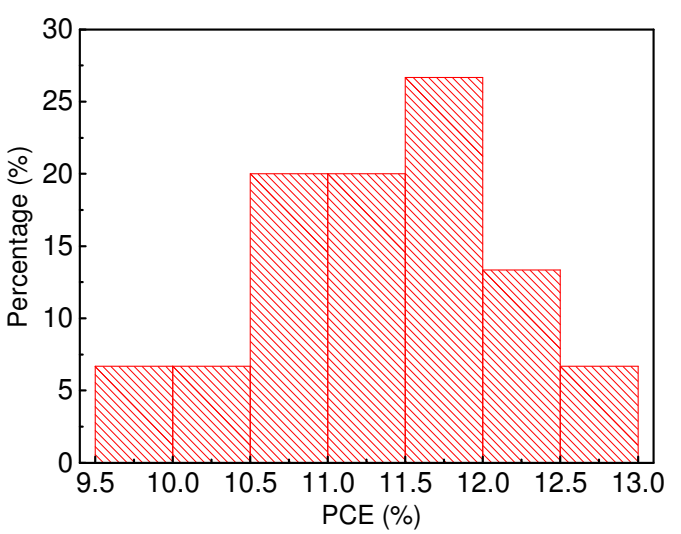

Figure 1. Y. Shao et al 
(a)

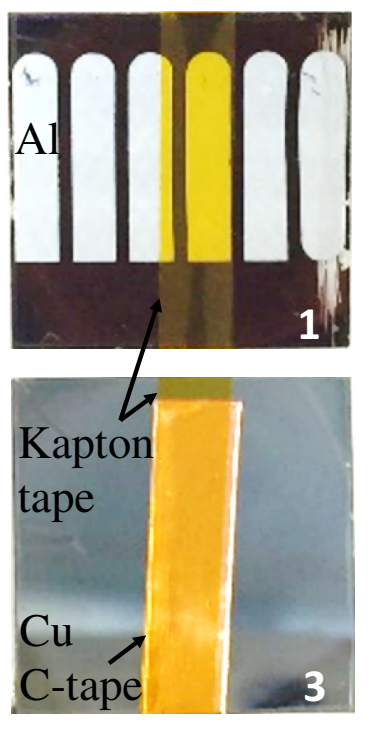

(b)
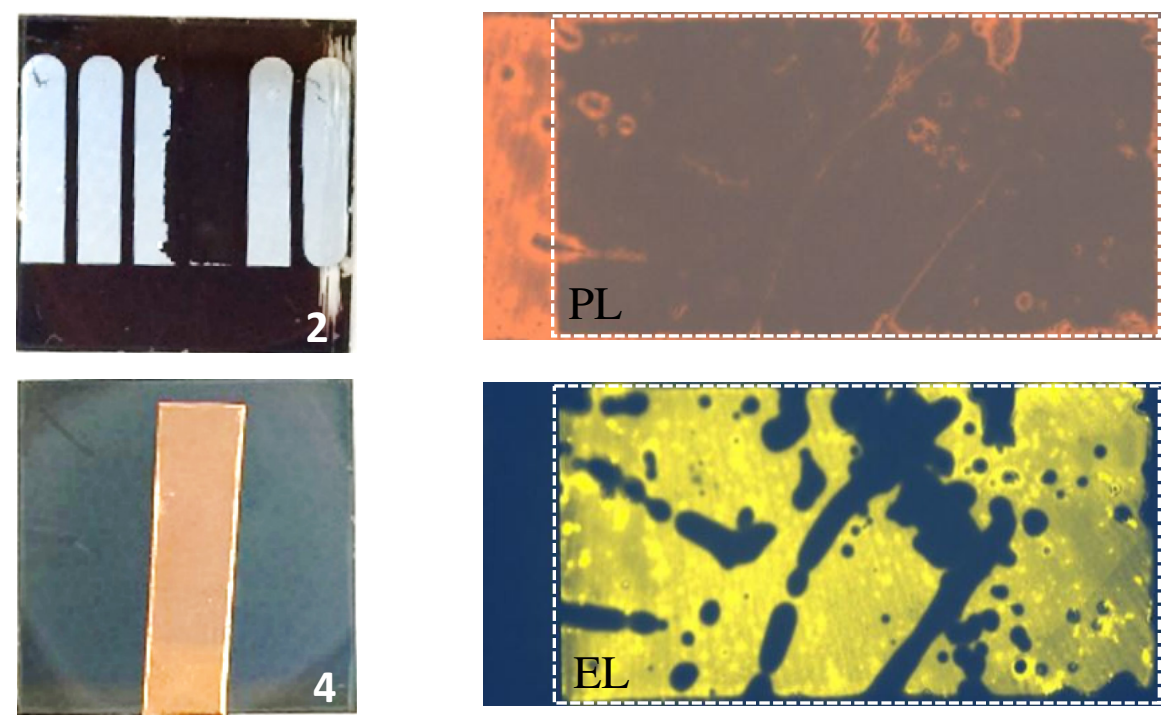

Figure 2 Y. Shao et al 
(a)

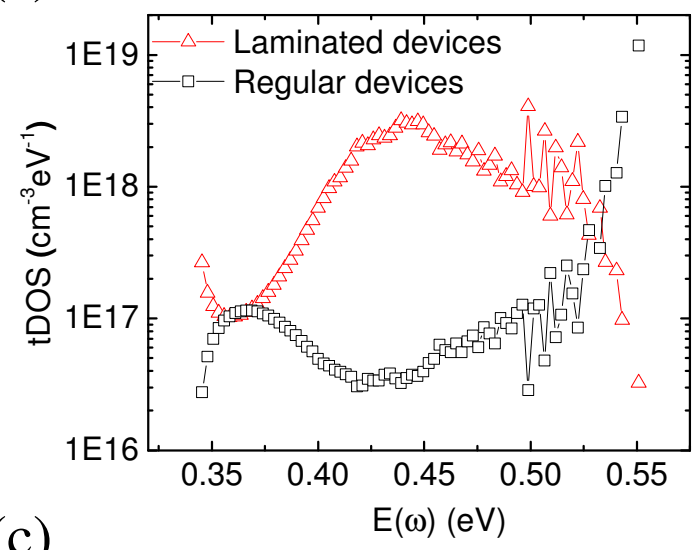

(c)

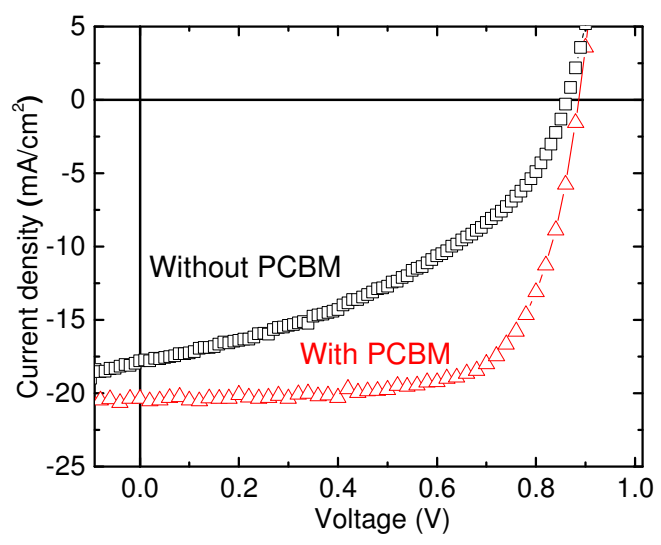

(b)

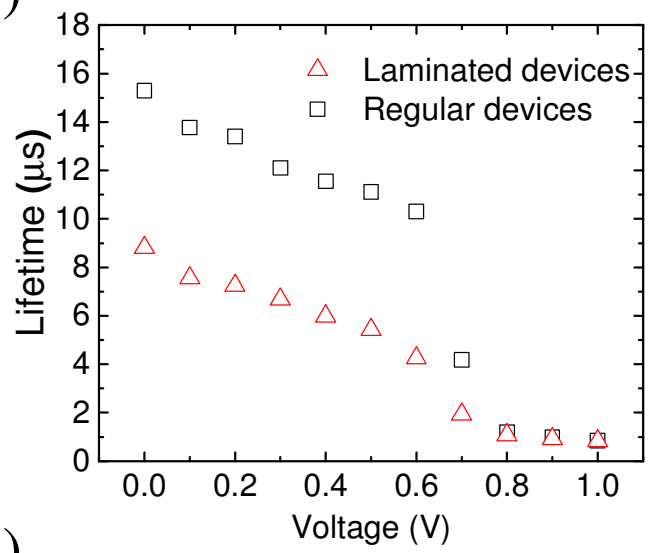

(d)

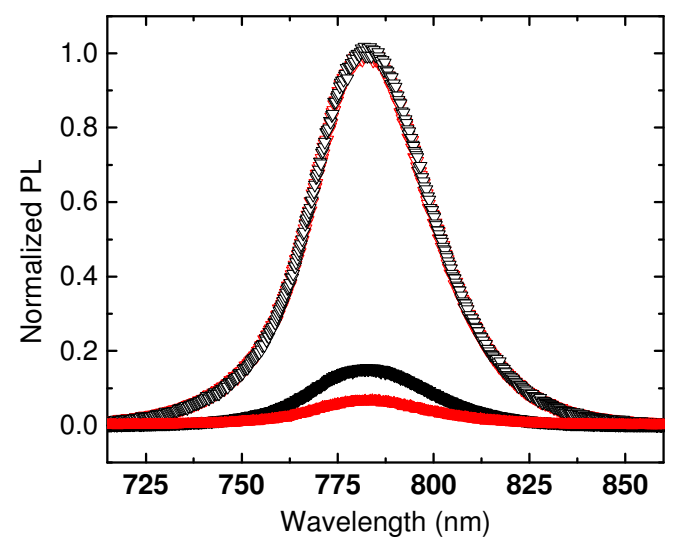

Figure 3. Y. Shao et al 
(a)

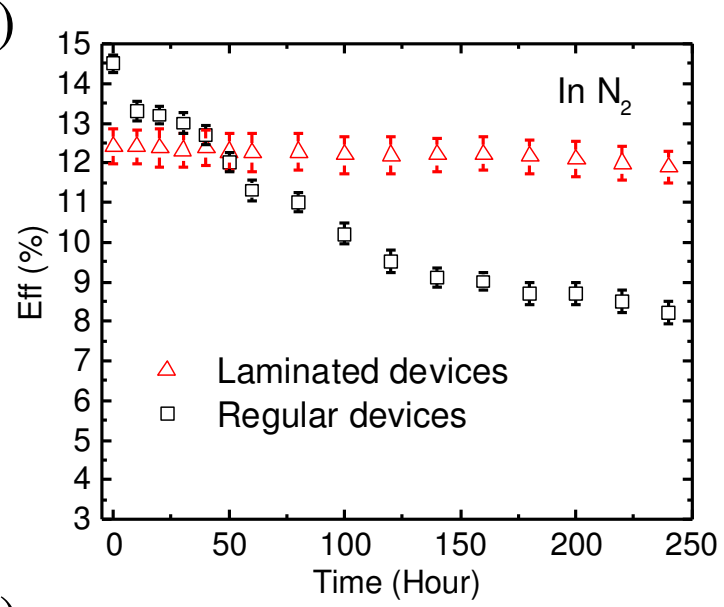

(b)

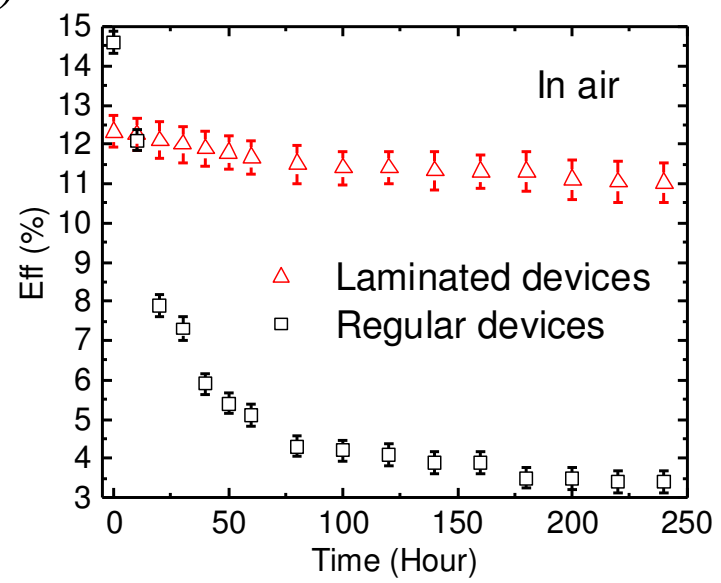

(c)

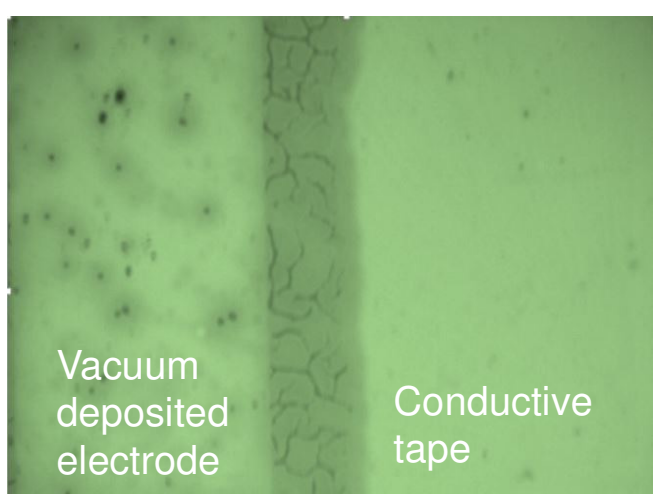

(d)

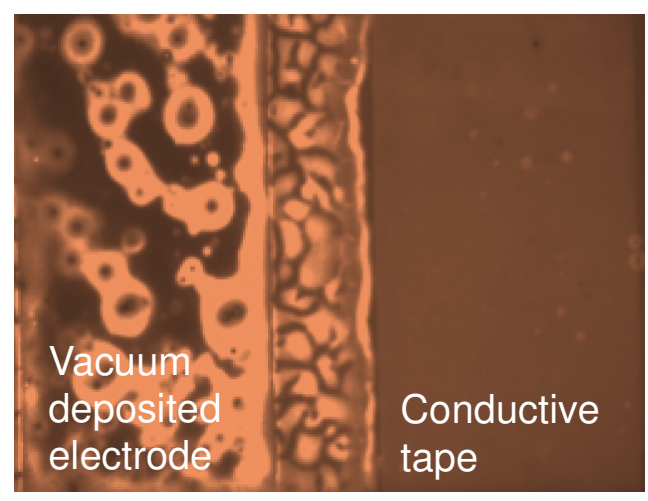

Figure 4. Y. Shao et al 

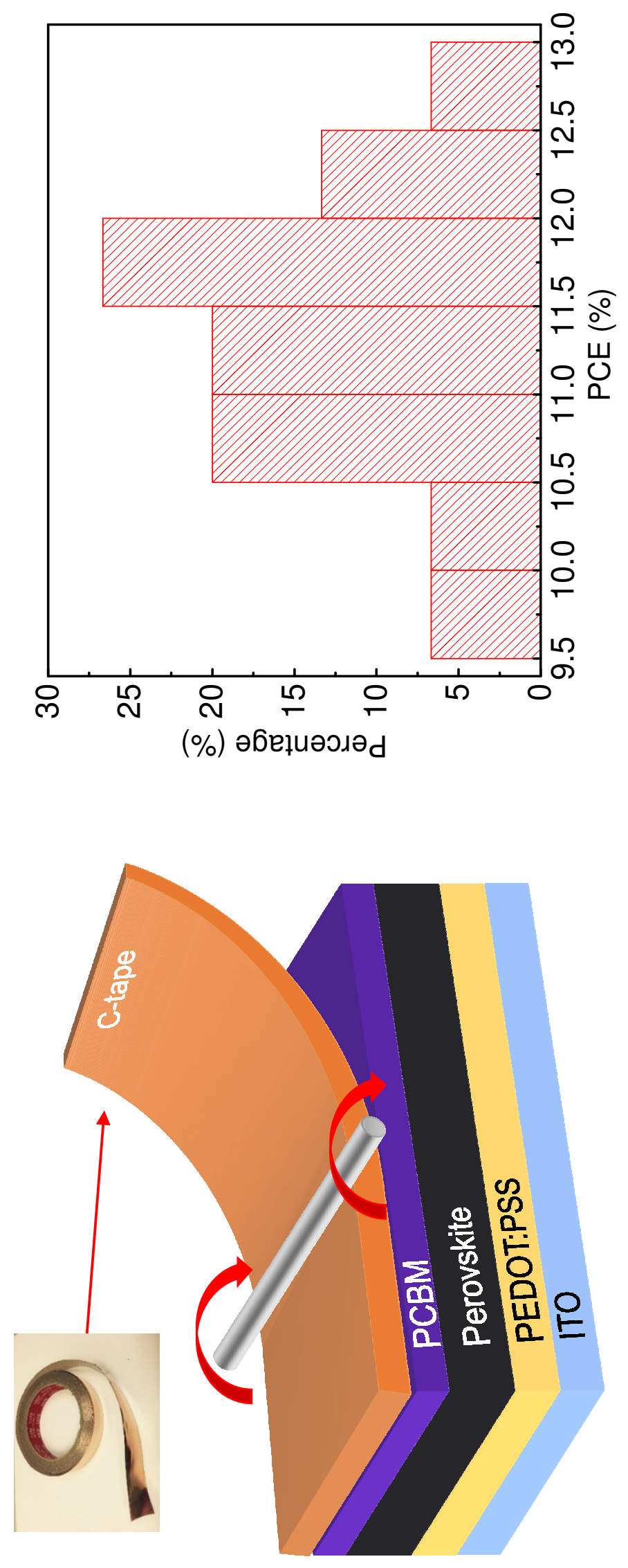